\title{
Left atrial appendage volume as a new predictor of atrial fibrillation recurrence after catheter ablation
}

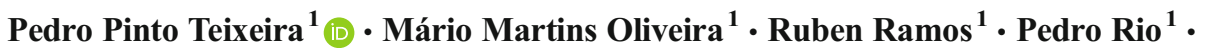 \\ Pedro Silva Cunha $^{1} \cdot$ Ana Sofia Delgado ${ }^{1} \cdot$ Ricardo Pimenta $^{1} \cdot$ Rui Cruz Ferreira $^{1}$
}

Received: 28 February 2017 / Accepted: 15 May 2017 / Published online: 31 May 2017

(C) Springer Science+Business Media New York 2017

\begin{abstract}
Purpose Recurrence of atrial fibrillation (AF) after catheter ablation is common, being clinically relevant to identify predictors of recurrence. The left atrial appendage (LAA) role as an $\mathrm{AF}$ trigger is scarcely explored. Our aim was to identify if LAA volume is an independent predictor of AF recurrence after catheter ablation.

Methods We analysed 52 patients (aged $54 \pm 10$ years, 58\% male) with paroxysmal and persistent $\mathrm{AF}$ who underwent a first AF catheter ablation and had performed contrast-enhanced cardiac computed tomography (CT) prior to the procedure.

Results The mean left atrial and LAA volumes measured by cardiac CT were $98.9 \pm 31.8$ and $9.3 \pm 3.5 \mathrm{~mL}$, respectively. All patients received successful pulmonary vein isolation and were followed up for 24 months. AF recurrence occurred in 17 patients $(33 \%)$. LAA volume was significantly greater in patients with $\mathrm{AF}$ recurrence than in those without recurrence $(11.3 \pm 2.9$ vs. $8.2 \pm 3.4 \mathrm{~mL} ; p=0.002)$. Multivariable analysis using Cox regression revealed that LAA volume (hazard ratio $1.32 ; 95 \%$ confidence interval $1.12-1.55 ; p=0.001$ ) and persistent $\mathrm{AF}$ (hazard ratio $4.22 ; 95 \%$ confidence interval $1.48-12.07 ; p=0.007$ ) were independent predictors for $\mathrm{AF}$ recurrence. An LAA volume greater than $8.825 \mathrm{~mL}$ predicted AF recurrence with $94 \%$ sensitivity and $66 \%$ specificity. The Kaplan-Meier analysis showed a lower rate free from AF recurrence in the group with an LAA volume $>8.825 \mathrm{~mL}$ $(p<0.001)$.
\end{abstract}

Pedro Pinto Teixeira

phpintoteixeira@gmail.com

1 Department of Cardiology, Hospital de Santa Marta, Centro Hospitalar de Lisboa Central, Rua de Santa Marta 50, 1169-024 Lisbon, Portugal
Conclusions Larger LAA volume was associated with AF recurrence after catheter ablation in patients with paroxysmal and persistent $\mathrm{AF}$.

Keywords Atrial fibrillation - Ablation · Left atrial appendage $\cdot$ Cardiac computed tomography

\section{Introduction}

The overall success rate of catheter ablation to treat atrial fibrillation $(\mathrm{AF})$ ranges from 60 to $90 \%$ [1-6]. To enhance the procedure's success rate, it is important to identify predictors of AF recurrence.

Some factors have been reported to have a role in predicting AF recurrence after ablation, namely AF duration, left atrial (LA) dimensions and cardiovascular comorbidities [6-10]. In this context, little is known about the left atrial appendage (LAA), which has an underrecognized role as an AF trigger despite being an acknowledged source of focal automaticity [11-15]. Di Biase et al. reported the LAA as a site of triggers and focal firing in $27 \%$ of 987 patients undergoing redo catheter AF ablation [16]. Recently, a randomized trial showed that electrical isolation of the LAA improved long-term freedom from atrial arrhythmias in patients with long-standing persistent AF submitted to catheter ablation [17]. However, the potential role of LAA volume in the prediction of AF recurrence has not yet been solved. LAA volume can be accurately measured using cardiac computed tomography $(\mathrm{CT})$, which is routinely performed before $\mathrm{AF}$ ablation for assessment of the LA and pulmonary vein anatomy and for image integration of the three-dimensional (3D) CTderived images with the real-time acquired electroanatomical mapping data during ablation [18-20]. 
The aim of this study was to explore the association between LAA volume and AF recurrence after catheter ablation in patients with paroxysmal and persistent AF.

\section{Methods}

We analysed patients with symptomatic paroxysmal and persistent $\mathrm{AF}$ who underwent a first $\mathrm{AF}$ catheter-based radiofrequency ablation in our centre between July 2013 and December 2014 and had performed cardiac CT prior to the procedure. Paroxysmal AF was characterized by episodes of AF that terminate spontaneously or with intervention within 7 days of onset. Persistent AF was defined as continuous AF that is sustained more than 7 days and long-standing persistent $\mathrm{AF}$ as continuous AF lasting more than 12 months [21].

Patients with incomplete circumferential pulmonary vein isolation and patients with inadequate image quality for LA and LAA volume measurements were excluded from the study.

\subsection{LAA volume assessed by cardiac computed tomography}

LAA volume was assessed from contrast-enhanced cardiac $\mathrm{CT}$ images acquired before the ablation, with the intent of studying the LA and pulmonary vein anatomy and providing $3 \mathrm{D}$ images to merge with the electroanatomical mapping during the procedure. Cardiac CT images were obtained using a 64-detector scanner (VCT LightSpeed, GE Healthcare ${ }^{\circledR}$ ) after administration of iodinated contrast agent $(90$ to $110 \mathrm{~mL}$ of Ultravist 370) at 4 to $5 \mathrm{~mL} / \mathrm{s}$ followed by 30 to $50 \mathrm{~mL}$ of normal saline at the same rate. Data were acquired using a retrospective ECG-controlled tube current modulation technique, where the highest tube current (450-500 mA) was applied only during the systolic phase of the cardiac cycle. Scanning was triggered once the contrast arrived at the ascending aorta and had higher intensity in the aorta than in the pulmonary trunk. Non-overlapped axial images were reconstructed from phase 20 to $50 \%$ of the R-R interval, with $5 \%$ interval increments and a slice thickness of $0.6 \mathrm{~mm}$. Image analysis was performed on a dedicated cardiac $\mathrm{CT}$ workstation (iNtuition, TeraRecon ${ }^{\circledR}$ ) with volume-rendering software. Maximal LAA and LA volumes were measured at the endsystolic phase (usually $40 \%$ of the R-R interval), just before the mitral valve opening, when the LA cavity was largest and the LV cavity was smallest. LA borders were traced on each axial slice, excluding the pulmonary veins and appendage at the ostia and using the mitral valve annulus as the landmark differentiating the LA from the left ventricle. Orthogonal views were used to accurately differentiate the LAA orifice from the LA. The LAA borders were traced manually on the axial slices, and LAA trabeculations were considered part of the LAA cavity. The volumes of the 3D LAA and LA were calculated by multiplying the area of each slice by its thickness and summing up all the volumes of each slice (Simpson method) (Fig. 1). Only patients with fully contrasted LA and LAA allowing for an accurate border delineation were included in the analysis.

Furthermore, we categorized the LAA shape into four different morphologies: cactus, chicken wing, windsock and cauliflower, as originally described by Wang et al. [22]. Briefly, the "cactus" is defined as a central lobe with one or more secondary lobes; "chicken wing" as a main lobe that bends from the proximal or middle part of the LAA; "windsock" as one dominant lobe with several secondary, or even tertiary, lobes; and "cauliflower" as limited overall length structure with complex irregular characteristics and often lacking a dominant lobe.

In addition to cardiac $\mathrm{CT}$, patients performed transthoracic echocardiogram with measurement of LA volume and diameter as well as left ventricular ejection fraction estimation.

\subsection{Electrophysiological study and catheter ablation procedure}

All patients underwent catheter-based circumferential pulmonary vein (PV) isolation using radiofrequency energy delivered by catheter with an open, irrigated tip. Three-dimensional electroanatomical mapping systems were used, and merging with CT-derived images was achieved. The endpoint of pulmonary vein isolation was the electrical isolation of all pulmonary veins from the LA, confirmed by entry and exit block assessed with the ablation catheter tip at four positions around the circumference of the PV ostia. Further linear ablation, including LA roof line and mitral isthmus line, was added at the discretion of the operator. Ablation of complex fractioned atrial electrograms (CFAEs) was also attempted at the discretion of the operator in cases where AF persisted after pulmonary vein isolation and linear ablation. If $\mathrm{AF}$ continued after the ablation, biphasic direct current shocks were applied to restore sinus rhythm.

\subsection{Follow-up and AF recurrence assessment}

All patients were followed up for 24 months after ablation. Assessment was performed at discharge and at the 3rd, 6th, 12th and 24th months by a clinic appointment, 12-lead ECG and 24-h Holter monitoring. In addition, patients who complained of palpitations or irregular pulse were instructed to seek immediate medical attention for prompt ECG recording. An external loop recorder was given to patients who developed symptoms suggestive of $\mathrm{AF}$ but had no documentation of AF on ECG or 24-h Holter monitoring. Oral anticoagulants were prescribed for a minimum of 6 months and eventually discontinued in case of a low thromboembolic score $\left(\mathrm{CHADS}_{2}\right.$ VASC score 0 or 1$)$. Antiarrhythmic drugs were 


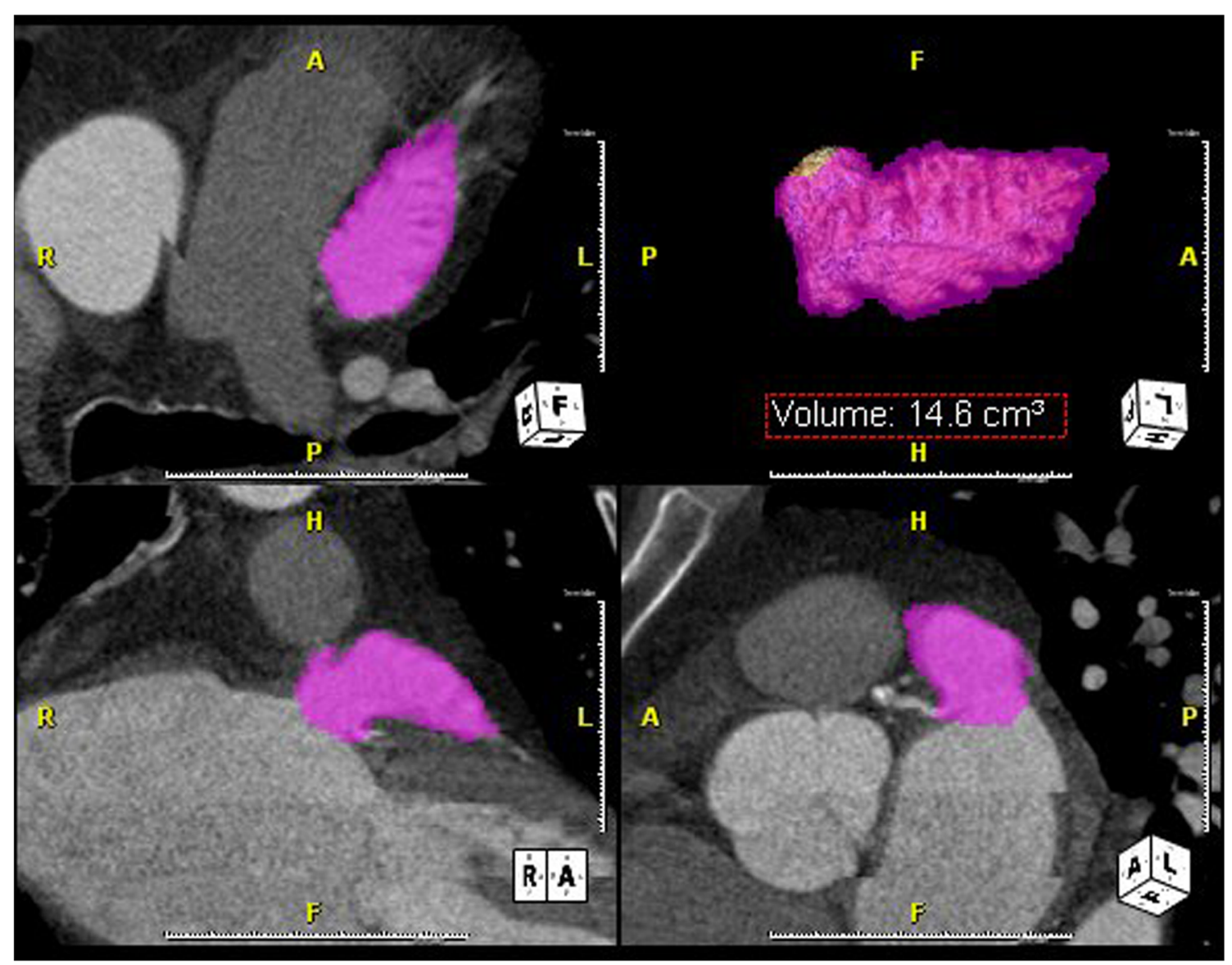

Fig. 1 Illustration of a large left atrial appendage (LAA) with a total volume of $14.6 \mathrm{~mL}$. The depicted 3D LAA was obtained after manual tracing of its borders on axial slices for volume measurement

continued for at least 6 months in patients who were taking them prior to the procedure, and were interrupted in case of no $\mathrm{AF}$ recurrence at 6 months of follow-up. AF recurrence was considered as any episode of AF (recorded by 12-lead ECG, 24-h Holter monitoring or external loop recorder) lasting $>30 \mathrm{~s}$ and occurring $>3$ months after ablation (blanking period) [23].

\subsection{Statistical analysis}

Continuous variables are presented as mean value \pm standard deviation and categorical variables as absolute numbers and relative frequencies. Student's $t$ test was performed to compare continuous variables, and a chi-square test was used to compare categorical variables. A Cox proportional hazards regression model was used to identify parameters associated with AF recurrence following catheter ablation. Variables with a $p$ value $<0.20$ in the univariate analysis were then considered in a multivariate analysis to determine any independent predictors associated with $\mathrm{AF}$ recurrence and calculate adjusted hazard ratios (HRs). LAA volume was further studied by using a receiver operating characteristic (ROC) curve to determine the optimal cut-off values for the prediction of $\mathrm{AF}$ recurrence. The best cutoff value was defined as the point combining the highest sensitivity and specificity. The Kaplan-Meier method estimated the event-free survival rate, and comparisons between different groups were rendered by a log-rank test. Statistical significance was accepted at a $p$ value $<0.05$. Statistical analysis was performed using SPSS Statistics, version 22.

Intraobserver variability and interobserver variability for LAA volume measurement were assessed using a randomly selected subset of 15 patients. Measurements were repeated by the same observer after an interval of $\geq 1$ week and by a second independent blinded observer. Reproducibility was estimated by intraclass correlation coefficient, with good agreement defined as $>0.80$.

\section{Results}

\subsection{Patients' characteristics}

During the study period, 78 patients underwent radiofrequency catheter ablation of paroxysmal or persistent AF. From these, 11 patients were excluded due to incomplete electrical PV isolation from the LA, 9 were excluded due to a lack of pre-procedural cardiac CT and 6 were excluded because of 
inadequate CT imaging with the LAA filling poorly with contrast. The final study population consisted of 52 patients (aged $54.4 \pm 9.7$ years, $58 \%$ male). AF recurrence occurred in 17 patients $(33 \%)$ during the 24-month follow-up, with a mean time until recurrence of $305.9 \pm 144.6$ days. Table 1 compares the baseline characteristics between patients with and without $\mathrm{AF}$ recurrence. Patients who had AF recurrence did not differ significantly with respect to age, gender, comorbidities (e.g. hypertension, coronary artery disease), LA volume or diameter, when compared to patients who did not experience recurrent AF. Circumferential pulmonary vein isolation was achieved in all patients. Additional linear ablation was performed in 14 patients, and the proportion of them who received this procedure was comparable to those with and without AF recurrence. The study sample included 30 patients with paroxysmal $\mathrm{AF}$ and 22 patients with persistent $\mathrm{AF}$, of whom seven had long-standing persistent AF. The recurrence rate was higher among patients with persistent $\mathrm{AF}$ ( 50 vs. $20 \%$ for patients with paroxysmal AF).

\subsection{LAA volume and imaging characteristics}

The mean LAA volume was $9.3 \pm 3.5 \mathrm{~mL}$. As depicted in Table 1 and Fig. 2, the LAA volume was significantly greater in patients with $\mathrm{AF}$ recurrence $(11.3 \pm 2.9$ vs. $8.2 \pm 3.4 \mathrm{~mL}$; $p=0.002$ ). This difference was notorious among the subgroup of patients with persistent AF: those who experienced AF recurrence had significantly larger LAA volume than those who did not $(11.5 \pm 2.2$ vs. $7.8 \pm 2.4 \mathrm{~mL} ; p=0.005)$. It is of note that the mean LA volume of patients with paroxysmal and persistent AF did not significantly differ $(94.1 \pm 33.8$ vs. $105.3 \pm 28.1 \mathrm{~mL} ; p=0.2$ ).

LAA volume did not correlate with gender $[9.8 \pm 3.4 \mathrm{~mL}$ (male) vs. $8.5 \pm 3.7 \mathrm{~mL}$ (female); $p=0.22]$, age $(r=0.08$, $p=0.58)$, body mass index $(r=0.15, p=0.29)$, left ventricular ejection fraction $(r=-0.01, p=0.92)$, persistent $\mathrm{AF}$ [9.4 $\pm 3.3 \mathrm{~mL}$ (with) vs. $8.9 \pm 4.2 \mathrm{~mL}$ (without); $p=0.46$ ] or hypertension $[9.7 \pm 2.9 \mathrm{~mL}$ (with) vs. $9.0 \pm 3.9 \mathrm{~mL}$ (without); $p=0.70]$. A good correlation was found between LAA volume and LA volume assessed by cardiac CT $(r=0.71, p<0.001)$. The mean LA volume derived from CT $(98.9 \pm 31.7 \mathrm{~mL})$ was larger than the mean LA volume measured by echocardiography $(89.4 \pm 28.0 \mathrm{~mL})$ but with a strong correlation between each other $(r=0.81, p<0.001)$.

Regarding LAA shape assessed by cardiac CT, the following morphologies were detected: cactus in 15 patients (29\%), chicken wing in $24(46 \%)$, windsock in $10(19 \%)$ and cauliflower in $3(6 \%)$. The distribution of LAA morphologies did
Table 1 Patients' characteristics by subgroup (no AF recurrence vs. $\mathrm{AF}$ recurrence)

\begin{tabular}{llll}
\hline & No AF recurrence & AF recurrence & $p$ value \\
\hline Clinical characteristics & & & \\
Age (years) & $54.1 \pm 10.9$ & $54.9 \pm 6.9$ & 0.77 \\
Male $(n, \%)$ & $19(54)$ & $11(65)$ & 0.56 \\
Body mass index $\left(\mathrm{kg} / \mathrm{m}^{2}\right)$ & $28.6 \pm 4.3$ & $27.8 \pm 4.1$ & 0.73 \\
Type of AF $(n, \%)$ & & & 0.03 \\
$\quad$ Paroxysmal AF & $24(69)$ & $6(35)$ & \\
$\quad$ Persistent AF & $11(31)$ & $11(65)$ & 0.11 \\
Hypertension $(n, \%)$ & $26(74)$ & $10(58)$ & 0.76 \\
Dyslipidaemia $(n, \%)$ & $19(54)$ & $1(6)$ & 0.47 \\
Diabetes mellitus $(n, \%)$ & $4(11)$ & $5(29)$ & 0.58 \\
Coronary artery disease $(n, \%)$ & $7(20)$ & $3(18)$ & 0.62 \\
Obstructive sleep apnoea $(n, \%)$ & $6(17)$ & & \\
Imaging parameters & & $11.3 \pm 2.9$ & 0.002 \\
Left atrial appendage volume $(\mathrm{mL})^{\mathrm{a}}$ & $8.2 \pm 3.4$ & $105.7 \pm 35.0$ & 0.28 \\
Left atrial volume $(\mathrm{mL})^{\mathrm{a}}$ & $95.5 \pm 30.0$ & $53.5 \pm 10.9$ & 0.64 \\
LV ejection fraction $(\%)^{\mathrm{b}}$ & $53.9 \pm 9.8$ & $43.9 \pm 10.9$ & 0.54 \\
${\text { Left atrial diameter }(\mathrm{mm})^{\mathrm{b}}}_{\text {Left atrial volume }(\mathrm{mL})^{\mathrm{b}}}$ & $43.8 \pm 10.9$ & $91.9 \pm 38.4$ & 0.81 \\
Ablation characteristics & $88.3 \pm 32.7$ & $6(35)$ & 0.59 \\
Additional linear ablation $(n, \%)$ & $8(23)$ & $2(12)$ & 0.57 \\
Additional CFAE ablation $(n, \%)$ & $4(11)$ & & \\
\hline
\end{tabular}

$A F$ atrial fibrillation, $C F A E$ complex fractioned atrial electrogram

${ }^{\text {a }}$ Assessed by cardiac CT

${ }^{\mathrm{b}}$ Assessed by echocardiography 


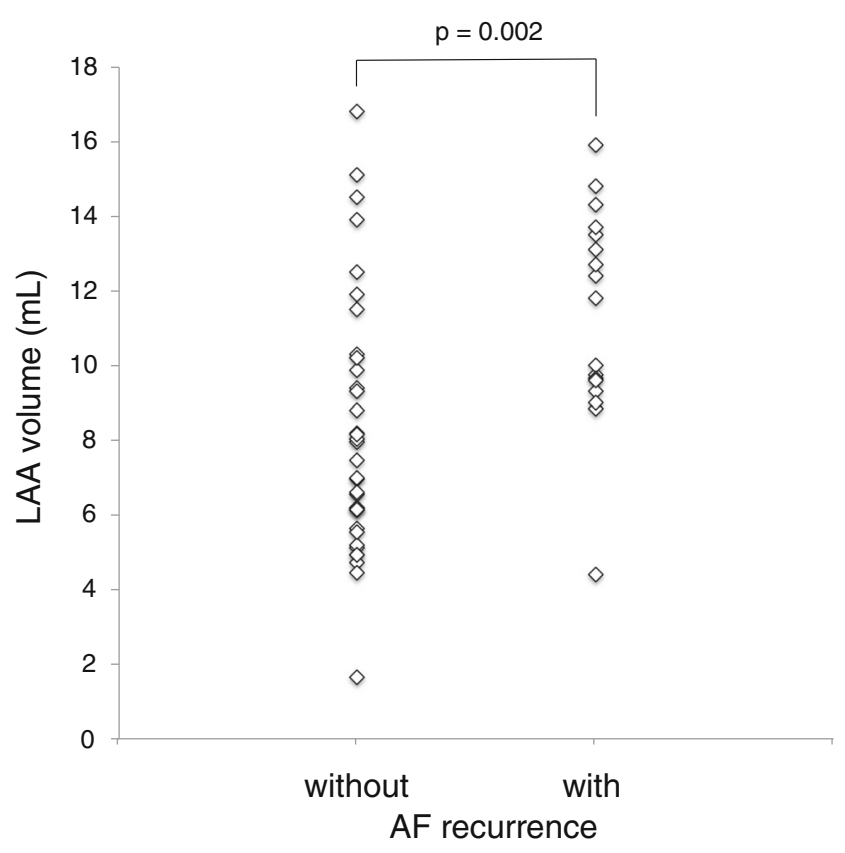

Fig. 2 Comparison of LAA volume between patients with and without $\mathrm{AF}$ recurrence. LAA volume was significantly larger in patients with $\mathrm{AF}$ recurrence than in those without $\mathrm{AF}$ recurrence $(11.3 \pm 2.9$ vs. $8.2 \pm 3.4 \mathrm{~mL} ; p=0.002)$

not significantly differ between patients with and without AF recurrence $(p>0.05)$. Moreover, no compelling correlation could be established between volume and distinct LAA morphologies $(p>0.05)$.

The intraclass correlation coefficient for LAA volume measurements was 0.95 for intraobserver variability and 0.89 for interobserver variability.

\subsection{LAA volume and prediction of AF recurrence}

To explore the associations between the various clinical parameters and AF recurrence, a Cox proportional hazards regression analysis was executed. In the univariate analysis, persistent AF, hypertension and LAA volume were linked to $\mathrm{AF}$ recurrence. In the multivariable analysis, LAA volume (adjusted HR 1.32, 95\% confidence interval 1.12-1.55; $p<0.001)$ remained as an independent predictor for AF recurrence, as well as persistent AF (adjusted HR 4.22, 95\% confidence interval 1.48-12.07; $p<0.001)$.

The ROC curve analysis revealed that an LAA volume $>8.825 \mathrm{~mL}$ was associated with AF recurrence after catheter ablation with a sensitivity of $94 \%$ and a specificity of $66 \%$ (area under the curve $=0.76$ ). In our study population, only one patient out of 24 with an LAA volume $<8.825 \mathrm{~mL}$ had AF recurrence. Furthermore, KaplanMeier curves demonstrated that patients with LAA volume above the $8.825 \mathrm{~mL}$ cut-off had a significantly higher rate of $\mathrm{AF}$ recurrence (Fig. 3).

\section{Discussion}

The main finding of our study was that a larger LAA volume was independently associated with AF recurrence after catheter ablation in patients with paroxysmal or persistent AF. To the best of our knowledge, this is the first study to describe such association.

Importantly, we found that among patients with persistent $\mathrm{AF}$, those who experienced recurrence had considerably higher LAA volumes. It is known that the outcomes of ablation in patients with persistent and long-standing persistent AF are poorer [1-4]. In these patients, many non-PV areas seem to be the source for initiation and maintenance of AF. The most common sites are the superior vena cava, the coronary sinus, the ligament of Marshall, the crista terminalis, the LA posterior wall and the LAA [16, 24-27]. In the recent BELIEF trial, Di Biase et al. showed improved freedom from atrial arrhythmias in patients with longstanding persistent AF who performed empirical electrical isolation of the LAA in addition to standard catheter ablation [17]. Although there is increasing evidence of the LAA importance as a trigger for atrial arrhythmias, the literature is scarce on the mechanisms and factors that may contribute to it. Previous studies have demonstrated the value of LAA flow velocity in the prediction of sinus rhythm maintenance after electrical cardioversion and, recently, Kanda et al. described an association between low LAA flow velocity and AF recurrence after catheter ablation in patients with persistent AF [28-30]. The current research suggests that LAA volume may be a surrogate of increased LAA arrhythmogenicity, similar to what happens with the LA volume: an enlarged LA is associated with electrical remodelling, which provides an arrhythmogenic substrate for AF triggers [31]. The LAA has a complex architecture with extensive pectinate muscles and highly heterogeneous orientation of the muscle bundles, which may facilitate slow conduction and block, along with the development of re-entry [11, 32, 33]. These electrical circuits may be heightened in the setting of an enlarged LAA and increased fibrosis.

It is accepted that cardiovascular comorbidities, such as hypertension and obesity, strongly influence atrial remodelling and enlargement [9, 34]. However, it is unclear what factors contribute to the enlargement of the LAA, which has distinct embryology, anatomy and histology from the LA [32, 33]. This may explain why some patients may have very large LAA with small- or moderately sized LA. Moreover, our finding that LAA volume, but not LA volume, was significantly greater in patients with AF recurrence compared to those without recurrence, reinforces the role of the LAA volume as an independent predictor for $\mathrm{AF}$ recurrence. It might become appropriate to empirically isolate the LAA in patients who present to catheter ablation with large LAA, especially those with persistent or long-standing persistent AF. In addition, LAA volume measurement by cardiac CT is feasible and readily accessible, given that patients programmed for catheter ablation should undergo the scan for procedure planning. 
Fig. 3 Kaplan-Meier analysis of time to AF recurrence. Study patients were divided into two groups based on the best cut-off value of LAA volume for prediction of AF recurrence. Patients with an LAA volume $>8.825 \mathrm{~mL}$ had a lower rate of freedom from AF recurrence
Kaplan-Meier analysis of time to AF recurrence

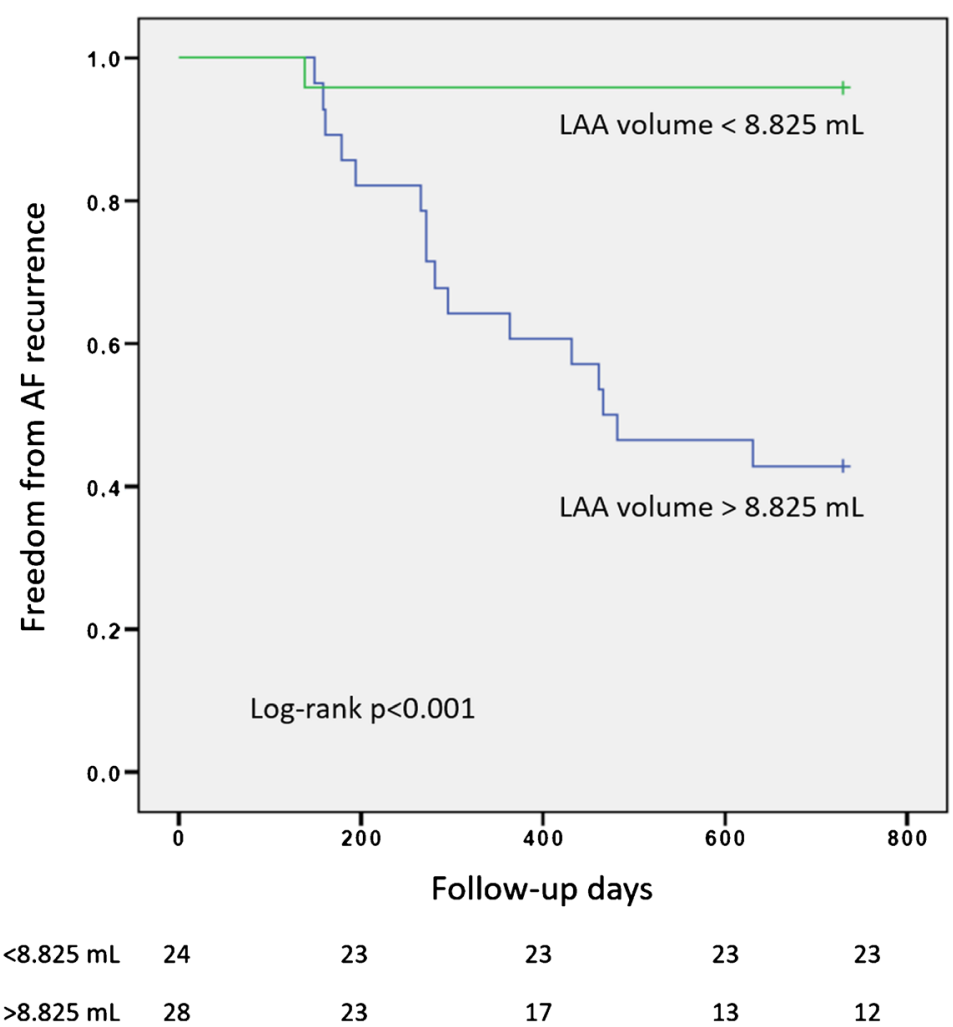

\subsection{Study limitations}

This was an exploratory study with important limitations. First, although we closely followed up patients with periodic clinic appointment, ECG and Holter monitoring, asymptomatic episodes of AF might not have been detected. Nonetheless, all patients included in our study had symptomatic AF before catheter ablation and, therefore, would probably seek medical attention if AF recurred. Second, the study design was retrospective and the sample size was modest; thus, we cannot rule out the bias of LA volume dependence. In addition, only a small number of patients had long-standing persistent AF. Those patients are probably the ones who might benefit more from LAA electrical isolation [17]. Prospective randomized studies are needed, particularly in the subgroup of patients with persistent and long-standing AF, to further clarify the clinical utility of LAA volume measurement as a predictor of AF recurrence and as a potential surrogate for electrical isolation of the LAA.

\section{Conclusions}

LAA volume, measured by cardiac CT, may be an independent predictor of AF recurrence in a population of patients with paroxysmal and persistent $\mathrm{AF}$ undergoing a first catheter ablation procedure. In patients with large LAA, the inclusion of empirical electrical isolation of the LAA might be appropriate. An LAA volume greater than $8.825 \mathrm{~mL}$ showed good accuracy to predict AF recurrence, with a sensitivity of $94 \%$ and a specificity of $66 \%$.

\section{Compliance with ethical standards}

Conflict of interest The authors declare that they have no conflict of interest.

\section{References}

1. Arbelo E, Brugada J, Hindricks G, et al. The atrial fibrillation ablation pilot study: a European survey on methodology and results of catheter ablation for atrial fibrillation conducted by the European Heart Rhythm Association. Eur Heart J. 2014;35:1466-78.

2. Ganesan AN, Shipp NJ, Brooks AG, et al. Long-term outcomes of catheter ablation of atrial fibrillation: a systematic review and metaanalysis. J Am Heart Assoc. 2013;2:e004549.

3. Tilz RR, Rillig A, Thum AM, et al. Catheter ablation of longstanding persistent atrial fibrillation: 5-year outcomes of the Hamburg Sequential Ablation Strategy. J Am Coll Cardiol. 2012:60:1921-9.

4. Scherr D, Khairy P, Miyazaki S, et al. Five-year outcome of catheter ablation of persistent atrial fibrillation using termination of atrial fibrillation as a procedural endpoint. Circ Arrhythm Electrophysiol. 2015;8:18-24. 
5. Verma A, Jiang CY, Betts TR, et al., STAR AF II Investigators. Approaches to catheter ablation for persistent atrial fibrillation. $\mathrm{N}$ Engl J Med 2015;372:1812-1822.

6. Bhargava M, Di Biase L, Mohanty P, et al. Impact of atrial fibrillation and repeat catheter ablation on long-term freedom from atrial fibrillation: results from a multicentre study. Heart Rhythm. 2009;6: 1403-12.

7. Helms AS, West JJ, Patel A, et al. Relation of left atrial volume from three-dimensional computed tomography to atrial fibrillation recurrence following ablation. Am J Cardiol. 2009;103:989-93.

8. Shin S, Park M, Jin W, et al. Left atrial volume is a predictor of atrial fibrillation recurrence after catheter ablation. J Am Soc Echocardiogr. 2008;21:697-702.

9. Park JH, Joung B, Son NH, Shim JM, Lee MH, Hwang C, et al. The electroanatomical remodelling of the left atrium is related to CHADS2/CHA2DS2VASc score and events of stroke in patients with atrial fibrillation. Europace. 2011;13:1541-9.

10. Berruezo A, Tamborero D, Mont L, et al. Pre-procedural predictors of atrial fibrillation recurrence after circumferential pulmonary vein ablation. Eur Heart J. 2007;28:836-41.

11. Krul SP, Berger WR, Smit NW, et al. Atrial fibrosis and conduction slowing in the left atrial appendage of patients undergoing thoracoscopic surgical pulmonary vein isolation for atrial fibrillation. Circ Arrhythm Electrophysiol. 2015;8:288-95.

12. Hocini M, Shah AJ, Nault I, et al. Localized reentry within the left atrial appendage: arrhythmogenic role in patients undergoing ablation of persistent atrial fibrillation. Heart Rhythm. 2011;8:1853-61.

13. Yamada T, Murakami Y, Yoshida Y, et al. Electrophysiologic and electrocardiographic characteristics and radiofrequency catheter ablation of focal atrial tachycardia originating from the left atrial appendage. Heart Rhythm. 2007;4:1284-91.

14. Takahashi Y, Sanders P, Rotter M, Haïssaguerre M. Disconnection of the left atrial appendage for elimination of foci maintaining atrial fibrillation. J Cardiovasc Electrophysiol. 2005;16:917-9.

15. Guo XG, Zhang JL, Ma J, et al. Management of focal atrial tachycardias originating from the atrial appendage with the combination of radiofrequency catheter ablation and minimally invasive atrial appendectomy. Heart Rhythm. 2014;11:17-25.

16. Di Biase L, Burkhardt JD, Mohanty P, et al. Left atrial appendage: an underrecognized trigger site of atrial fibrillation. Circulation. 2010;122:109-18.

17. Di Biase L, Burkhardt JD, Mohanty P, et al. Left atrial appendage isolation in patients with longstanding persistent $\mathrm{AF}$ undergoing catheter ablation: BELIEF trial. J Am Coll Cardiol. 2016;68: 1929-40.

18. Tops LF, Bax JJ, Zeppenfeld K, Jongbloed MR, Lamb HJ, van der Wall EE, et al. Fusion of multislice computed tomography imaging with three-dimensional electroanatomic mapping to guide radiofrequency catheter ablation procedures. Heart Rhythm. 2005;2:1076-81.

19. Taina M, Korhonen M, Haataja M, et al. Morphological and volumetric analysis of left atrial appendage and left atrium: cardiac computed tomography-based reproducibility assessment. PLoS One. 2014;9:e101580.

20. To AC, Flamm SD, Marwick TH, Klein AL. Clinical utility of multimodality LA imaging: assessment of size, function, and structure. JACC Cardiovasc Imaging. 2011;4:788-98.

21. January CT, Wann LS, Alpert JS, et al. 2014 AHA/ACC/HRS guideline for the management of patients with atrial fibrillation: a report of the American College of Cardiology/American Heart Association Task Force on Practice Guidelines and the Heart Rhythm Society. J Am Coll Cardiol. 2014;64:e1-76.

22. Wang Y, Di Biase L, Horton RP, et al. Left atrial appendage studied by computed tomography to help planning for appendage closure device placement. J Cardiovasc Electrophysiol. 2010;21:973-82.

23. Oliveira M. How to follow atrial fibrillation ablation patients? J Atr Fibrillation. 2014;7(3):1087.

24. Tsai CF, Tai CT, Hsieh MH, et al. Initiation of atrial fibrillation by ectopic beats originating from the superior vena cava: electrophysiological characteristics and results of radiofrequency ablation. Circulation. 2000;102:67-74.

25. Katritsis D, Ioannidis JP, Anagnostopoulos CE, et al. Identification and catheter ablation of extracardiac and intracardiac components of ligament of Marshall tissue for treatment of paroxysmal atrial fibrillation. J Cardiovasc Electrophysiol. 2001;12:750-8.

26. Hwang C, Wu TJ, Doshi RN, et al. Vein of Marshall cannulation for the analysis of electrical activity in patients with focal atrial fibrillation. Circulation. 2000;101:1503-8.

27. Nademanee K, McKenzie J, Kosar E, et al. A new approach for catheter ablation of atrial fibrillation: mapping of the electrophysiologic substrate. J Am Coll Cardiol. 2004;43:2044-53.

28. Antonielli E, Pizzuti A, Palinkas A, Tanga M, Gruber N, Michelassi $\mathrm{C}$, et al. Clinical value of left atrial appendage flow for prediction of long-term sinus rhythm maintenance in patients with nonvalvular atrial fibrillation. J Am Coll Cardiol. 2002;39:1443-9.

29. Palinkas A, Antonielli E, Picano E, et al. Clinical value of left atrial appendage flow velocity for predicting of cardioversion success in patients with non-valvular atrial fibrillation. Eur Heart J. 2001;22: 2201-8.

30. Kanda T, Masuda M, Sunaga A, et al. Low left atrial appendage flow velocity predicts recurrence of atrial fibrillation after catheter ablation of persistent atrial fibrillation. J Cardiol. 2015;66:377-81.

31. Kojodjojo P, Peters NS, Davies DW, Kanagaratnam P. Characterization of the electroanatomical substrate in human atrial fibrillation: the relationship between changes in atrial volume, refractoriness, wavefront propagation velocities, and AF burden. $\mathrm{J}$ Cardiovasc Electrophysiol. 2007;18:269-75.

32. Cabrera JA, Ho SY, Climent V, Sanchez-Quintana D. The architecture of the left lateral atrial wall: a particular anatomic region with implications for ablation of atrial fibrillation. Eur Heart J. 2008;29: 356-62.

33. Ho SY, Cabrera JA, Sanchez-Quintana D. Left atrial anatomy revisited. Circ Arrhythm Electrophysiol. 2012;5:220-8.

34. Wang TJ, Parise H, Levy D, et al. Obesity and the risk of new-onset atrial fibrillation. JAMA. 2004;292:2471-7. 